\title{
Initiation of $\mathrm{V}(\mathrm{D}) \mathrm{J}$ recombination in vivo: role of recombination signal sequences in formation of single and paired double-strand breaks
}

\author{
Sharri Bockheim Steen, Larissa Gomelsky, \\ Silvia L.Speidel ${ }^{1}$ and David B.Roth ${ }^{1,2}$
}

Program in Cellular and Molecular Biology and ${ }^{1}$ Department of Microbiology and Immunology, Baylor College of Medicine, Houston, TX 77030, USA

${ }^{2}$ Corresponding author

In $\mathrm{V}(\mathrm{D}) \mathrm{J}$ recombination, double-strand breaks (DSBs) are introduced at recombination signal sequences (RSSs) which consist of three distinct elements: a heptamer, a 12 or 23 nucleotide spacer and a nonamer. Efficient DSB formation requires a 12/23 RSS pair and occurs at both RSS in a temporally coupled fashion (coupled cleavage). It remains unknown which RSS elements are important for coupled cleavage. Furthermore, it has not been established whether some RSS components are critical only for cleavage in cis, with others mainly promoting cleavage in trans at the partner RSS. We investigated these questions by analyzing the effects of RSS mutations on the formation of DSBs in vivo. The abundance of DSBs in cis (at the mutant RSS) and in trans (at the consensus RSS) was determined using an established ligation-mediated PCR assay. We also developed a Southern blotting approach that allows the first direct measurement of dual and single RSS cleavage in vivo. Our results demonstrate that the heptamer, spacer and nonamer elements are all required for coupled cleavage in vivo. These studies also provide evidence for cleavage events involving a single RSS both in mutant substrates and in substrates containing a consensus 12/23 RSS pair.

Keywords: double-strand break/RAG/recombination signal sequence/synapsis/V(D)J recombination

\section{Introduction}

The vertebrate immune system produces an extensive repertoire of immunoglobulins and T cell receptors capable of engaging a wide variety of distinct foreign antigens. This diversity of receptor specificities is generated by $\mathrm{V}(\mathrm{D}) \mathrm{J}$ recombination, which assembles the exons encoding the antigen binding domains from separate $\mathrm{V}$ (variable), $\mathrm{D}$ (diversity) and $\mathrm{J}$ (joining) gene segments (for recent review, see Bogue and Roth, 1996). These so-called coding segments are flanked by conserved recombination signal sequences (RSSs), which consist of three elements: a highly conserved heptamer, a 12 or 23 nucleotide 'spacer' and a conserved, AT-rich nonamer (Figure 1A). Efficient recombination only occurs between a 12-RSS and a 23RSS, an important regulatory requirement known as the '12/23 rule' (Lewis, 1994).

Recombination involves introduction of a double-strand break (DSB) between the RSS heptamer and the adjacent coding segment, generating two types of DNA terminus: blunt signal ends and covalently sealed coding ends, which are intermediates in the reaction and join to form a signal joint and a coding joint (Roth et al., 1992a,b; Schlissel et al., 1993; Ramsden and Gellert, 1995; Zhu and Roth, 1995). Experiments using cell-free systems have established that RAG-1 and RAG-2, the products of the recombination activating genes (Schatz et al., 1989; Oettinger et al., 1990), are the only protein factors necessary for proper DSB formation at an RSS (McBlane et al., 1995).

The precise roles of the components of the RSS in the cleavage reaction remain undefined. Mutational analysis has shown that the heptamer, spacer and nonamer elements are all important for efficient recombination in vivo (Hesse et al., 1989; Akamatsu et al., 1994). While these studies provided important information about the requirements for recombination under physiological conditions, the experimental systems used measured only completed recombination events and could not assess effects on the individual cleavage and joining steps.

More recently, the cleavage step has been studied in cell-free systems. The behavior of this reaction is dramatically affected by the incubation conditions. In the presence of $\mathrm{Mg}^{2+}$, efficient cleavage requires a 12/23 RSS pair and generally occurs at both RSSs in a temporally coupled fashion (termed coupled cleavage) (Eastman et al., 1996; van Gent et al., 1996). These data suggest that under these reaction conditions DSB formation may occur in the context of a synaptic complex involving a 12/23 RSS pair, an interpretation that is supported by analysis of several substrates bearing RSS mutations (Eastman et al., 1996). However, in the presence of $\mathrm{Mn}^{2+}$, cleavage at the two RSSs is uncoupled, and substrates containing a single RSS are cleaved efficiently (van Gent et al., 1995, 1996; Eastman et al., 1996). Thus, cleavage under these 'relaxed' conditions does not require formation of a normal synaptic complex.

The use of relaxed conditions facilitated detailed examination of the requirements for DSB formation at an isolated RSS by allowing analysis of cleavage using a number of short oligonucleotide substrates containing a single, mutationally altered RSS (Cuomo et al., 1996; Ramsden et al., 1996). These studies provided the first clues that the individual components of the RSS perform different functions and suggest that the nonamer plays an important role in binding of the RAG proteins, while the heptamer is important for the chemical steps of cleavage (Ramsden et al., 1996). However, the use of $\mathrm{Mn}^{2+}$ precluded analysis of the effects of RSS mutations on coupled cleavage. Furthermore, the observation that $\mathrm{Mn}^{2+}$ promotes bypass of the $12 / 23$ rule raises the possibility that this ion might influence the properties of the cleavage 

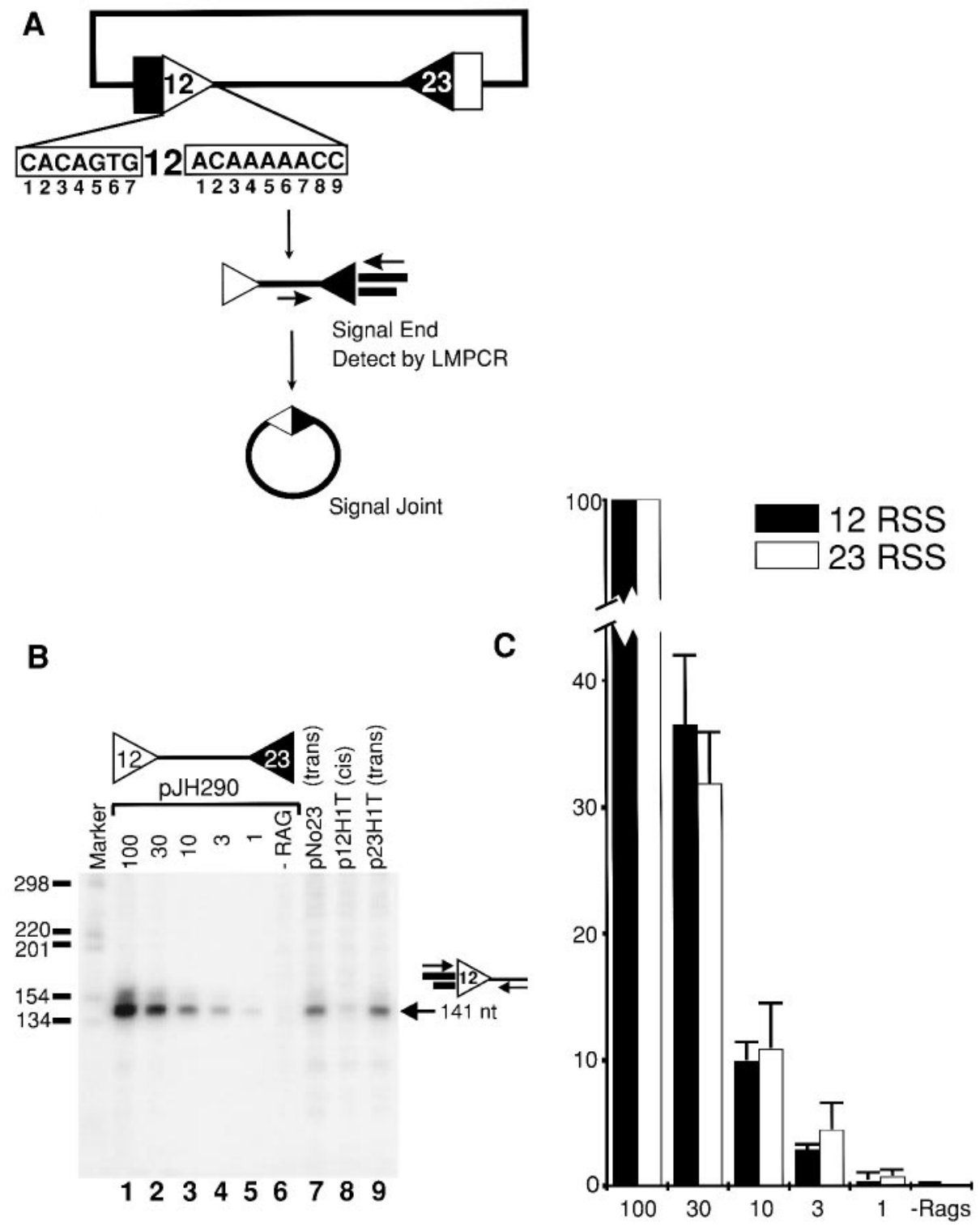

Fig. 1. Ligation-mediated PCR (LMPCR) analysis of DSB. (A) A schematic diagram of the recombination substrate pJH290 showing the heptamer and nonamer sequences of one RSS. A pair of annealed oligonucleotides (thick bars) is ligated to signal ends present in DNA harvested from transient transfection of a recombination substrate and RAG expression vectors. PCR is performed using appropriate primers (arrows). Symbols: open triangle, 12 spacer RSS; shaded triangle, 23 spacer RSS; squares, coding segments. (B) A representative blot showing LMPCR analysis of DSB at the 12-RSS. Lanes 1-5 contain a series of 3-fold dilutions of DNA from transfection of pJH290 that was added to the ligation reaction (100 refers to undiluted DNA; 30 refers to $30 \%$ of the amount of DNA in 100, etc.). Lane 6 contains undiluted PCR products from a transfection of pJH290 in the absence of RAG expression vectors; lanes 7-9 contain undiluted PCR products from transfections of the indicated mutant substrates. The blot was probed with a ${ }^{32} \mathrm{P}$ end-labeled oligonucleotide (DR55). (C) PhosphorImager quantitation of PCR products derived from dilution series, including lanes 1-6 of the blot in (B). The average and standard deviations are based on comparison with the undiluted DNA level on each blot and were derived from three independent transfections.

reaction in other, unknown ways which could affect the interpretation of these results.

More generally, it is not known whether the current in vitro reaction conditions, which employ purified, truncated RAG proteins (for the sake of solubility) and naked DNA substrates, faithfully recapitulate the characteristics of the cleavage reaction as it occurs in living cells. For example, cleavage at isolated RSS has been observed in both $\mathrm{Mn}^{2+}$ and $\mathrm{Mg}^{2+}$, although it is much more prevalent under the former conditions. The physiological relevance of this single RSS cleavage activity remains unclear. Additionally, in both $\mathrm{Mn}^{2+}$ and $\mathrm{Mg}^{2+}$ DSB formation is substantially more efficient at the 12-RSS than at the 23-
RSS (van Gent et al., 1996). It is not known whether this behavior is an intrinsic property of the normal reaction or merely reflects the need for further optimization of the in vitro incubation conditions. Here we have addressed these and related questions by analyzing cleavage in vivo.

We recently described a system that employs ligationmediated PCR (LMPCR) to detect and quantify cleaved $\mathrm{V}(\mathrm{D}) \mathrm{J}$ recombination intermediates generated in vivo from plasmid substrates (Steen et al., 1996). Here we present the first analysis of the effects of point mutations in the RSS on the cleavage step of $\mathrm{V}(\mathrm{D}) \mathrm{J}$ recombination in vivo. We examined the roles of the heptamer, spacer and nonamer elements in DSB formation in living cells. 
Table I. Recombination substrates used in this study

\begin{tabular}{|c|c|c|c|c|}
\hline & Plasmid & Working name & 12-RSS & 23-RSS \\
\hline \multirow[t]{4}{*}{ Controls } & pJH290 & pJH290 & consensus & consensus \\
\hline & pNoRSS & $\mathrm{pJH} 452$ & none & none \\
\hline & No23 & pGL1 & consensus & none \\
\hline & pNo12 & pGL2 & none & consensus \\
\hline \multirow[t]{11}{*}{ Heptamer } & pJH290 & pJH290 & CACAGTG & CACAGTG \\
\hline & $\mathrm{p} 12 \mathrm{H} 1 \mathrm{~T}$ & pSS1 & tACAGTG & CACAGTG \\
\hline & $\mathrm{p} 12 \mathrm{H} 1 \mathrm{~A}$ & pJH540 & aACAGTG & CACAGTG \\
\hline & $\mathrm{p} 12 \mathrm{H} 1 \mathrm{G}$ & pJH541 & gACAGTG & CACAGTG \\
\hline & $\mathrm{p} 23 \mathrm{H} 1 \mathrm{~T}$ & pJH315 & CACAGTG & tACAGTG \\
\hline & $\mathrm{p} 23 \mathrm{H} 3 \mathrm{~T}$ & pJH363 & CACAGTG & CAtAGTG \\
\hline & $\mathrm{p} 23 \mathrm{H} 4 \mathrm{~T}$ & pJH365 & CACAGTG & CACtGTG \\
\hline & $\mathrm{p} 23 \mathrm{H} 5 \mathrm{~A}$ & pJH367 & CACAGTG & CACAaTG \\
\hline & $\mathrm{p} 23 \mathrm{H} 7 \mathrm{~A}$ & pJH385 & CACAGTG & CACAGTa \\
\hline & $\mathrm{p} 12 \mathrm{H} 7 \mathrm{~A} / 23 \mathrm{H} 7 \mathrm{~A}$ & pJH397 & CACAGTa & CACAGTa \\
\hline & $\mathrm{p} 12 \mathrm{H} 7 \mathrm{~A} / 23 \mathrm{H} 1 \mathrm{~T}$ & pJH383 & CACAGTa & tACAGTG \\
\hline \multirow[t]{4}{*}{ Nonamer } & pJH290 & pJH290 & АСАААAACC & АСАААAАCC \\
\hline & $\mathrm{p} 23 \mathrm{~N} 6 \mathrm{G}$ & pJH432 & ACAAAAACC & ACAAAgACC \\
\hline & p12N8T9A & pDR11 & ACAAAAAta & ACAAAAACC \\
\hline & $\mathrm{p} 12 \mathrm{~N} 5 \mathrm{G} / 23 \mathrm{~N} 5 \mathrm{G}$ & pJH442 & ACAAgAACC & ACAAgAACC \\
\hline \multirow[t]{5}{*}{ Spacer } & pJH290 & pJH290 & consensus & consensus \\
\hline & $\mathrm{p} 23 \mathrm{~S}+1$ & pJH389 & plus 1 & consensus \\
\hline & $\mathrm{p} 23 \mathrm{~S}-2$ & pJH319 & consensus & minus 2 \\
\hline & p23S-3 & pJH321 & consensus & minus 3 \\
\hline & p12S-3/23S-3 & pJH327 & minus 3 & minus 3 \\
\hline \multirow[t]{2}{*}{ Multiple } & p12mult & pLG1 & multiple $^{\mathrm{a}}$ & consensus \\
\hline & p23mult & pJH430 & consensus & multiple ${ }^{b}$ \\
\hline
\end{tabular}

Plasmid names used in this study are listed in the first column; working designations are listed in the second column.

In the 12-RSS of p12mult, the fourth position of the heptamer has been changed to $\mathrm{T}$ and the eighth and ninth positions of the nonamer have been changed to $\mathrm{T}$ and $\mathrm{A}$, respectively.

${ }^{\mathrm{b}}$ In the 23-RSS of p23mult, the sixth and seventh positions of the heptamer have been changed to G and T, respectively, the spacer contains one additional nucleotide, and the fifth position of the nonamer has been changed to $\mathrm{G}$.

Specifically, we explored the possibility that some elements might be required only for DSB formation at a single RSS, with others more important for coordination of cleavage at a 12/23 RSS pair, a hypothesis which has precedents in DNA transposition reactions (Sakai et al., 1995). Furthermore, we investigated whether the RSS might play a role in the joining reaction by comparing the effects of RSS mutations on DSB formation and on the efficiency of recombination.

Our results show that most cleavage events involve an RSS pair, and that efficient DSB formation in vivo requires all three elements (heptamer, spacer and nonamer) of both RSSs. These data provide important in vivo support for the hypothesis that formation of a synaptic complex involving a 12/23 RSS pair is a prerequisite for efficient cleavage. However, a consensus RSS paired with a wide variety of severely defective RSSs is still cleaved at low levels. Furthermore, Southern blotting experiments revealed similar, low levels of single RSS cleavage even in substrates containing a consensus 12/23 RSS pair. The data provide an in vivo correlate for the single RSS cleavage events observed in cell-free systems and demonstrate that under physiological conditions the cleavage mechanism can, infrequently, bypass the requirement for a 12/23 RSS pair.

\section{Results}

\section{Experimental design}

A number of laboratories have shown that, when transfected with expression vectors encoding RAG-1 and
RAG-2, fibroblasts efficiently carry out V(D)J rearrangement of plasmid recombination substrates (Oettinger et al., 1990; Kallenbach et al., 1992; Sadofsky et al., 1993, 1994; Silver et al., 1993; Cuomo and Oettinger, 1994; Kirch et al., 1996). We have demonstrated recently that accurate formation of DSBs, regulated by the 12/23 rule, occurs in this system (Steen et al., 1996). The signal ends generated by cleavage of these substrates are full-length and blunt (Steen et al., 1996), as observed in endogenous $\mathrm{T}$ cell receptor and immunoglobulin loci in developing lymphocytes (Roth et al., 1993; Schlissel et al., 1993; Zhu and Roth, 1995). Particular advantages of this approach include consistent generation of high levels of signal ends which facilitates detection of rare events (see below) and the ability to study a wide variety of mutant substrates. In addition, either full-length or truncated versions of the RAG proteins can be used, allowing direct comparison with in vitro systems which employ truncated RAG proteins. Of course, higher order regulation of rearrangement, such as changes in locus accessibility during lymphocyte development, is not recapitulated in this system.

Plasmid recombination substrates containing one consensus RSS and one RSS with alterations in the heptamer, spacer or nonamer elements were introduced into cultured fibroblasts along with expression vectors encoding RAG-1 and RAG-2. DSB formation in cis (at the mutated RSS) and in trans (at the consensus partner RSS) was measured using a quantitative LMPCR assay (Steen et al., 1996) or by Southern blotting.

The plasmid substrates analyzed in this study are listed in Table I. All substrates are based on the plasmid pJH290 
(Figure 1A). Substrate nomenclature reflects the nature of the RSS mutation(s). For example, in p12H1T the first nucleotide in the heptamer of the 12-RSS was replaced by a $\mathrm{T}$ residue; in $\mathrm{p} 23 \mathrm{~S}-2$, the 23 -RSS spacer was shortened by two nucleotides; in pNo12, the 12-RSS was removed.

For each substrate, the abundance of signal ends was determined by comparison with a dilution series derived from a parallel transfection of $\mathrm{pJH} 290$. A representative experiment demonstrating analysis of DSBs at the 12RSS is shown in Figure 1B. As expected, no DSBs were observed when pJH290 was transfected in the absence of RAG-1 and RAG-2 (lane 6). Quantitation of the PCR products derived from the dilution series in this (lanes 1-5) and two other independent transfections reveals that the abundance of PCR products derived from both 12 and 23 signal ends is reproducibly proportional to the amount of DNA assayed over at least a 100-fold range (Figure 1C). Previous work has shown that this PCR assay detects 12 and 23 ends with similar sensitivity (Steen et al., 1996).

\section{Effects of RSS mutations on DSB formation in cis and in trans}

Substrates with mutations in the heptamer, spacer and nonamer elements were analyzed to determine their effects on DSB formation. Representative results from three mutant substrates are shown in Figure 1B. As shown previously (Steen et al., 1996), a substrate with only one RSS yields low levels of DSBs (pNo23; Figure 1B, lane 7). Analysis of signal ends formed by cleavage at the 12RSS of p12H1T demonstrates that this mutation severely reduces DSB formation in cis ( $<1 \%$ of pJH290; lane 8$)$. However, analysis of the consensus 12-RSS of p23H1T (lane 9) illustrates that this same heptamer mutation also affects DSB formation in trans (at the consensus partner RSS) although much less severely ( 10\% of pJH290; similar to pNo23).

Substrates were tested in at least three independent transfections, and the data are summarized in Figure 2, which displays the average abundance of DSB in cis and in trans, normalized to levels of DSB observed using pJH290 in each transfection. As expected, DSBs were not observed in a substrate with no RSSs or when substrates were transfected in the absence of RAG expression vectors (Figure 2A).

We initially tested substrates containing mutations in the heptamer positions adjacent to the cleavage site, reasoning that these positions are likely to be critically important for cleavage in cis. Three different alterations in the first nucleotide of the heptamer, which is directly involved in the chemical steps of DSB formation, were tested. As shown in Figure 2A, all three mutations (p12H1T, p12H1A and p12H1G) virtually abolished DSB formation in cis. Identical results were obtained with a substrate containing a mutation in the first position of the heptamer of the 23-RSS (p23H1T). Alteration of the third position ( $\mathrm{p} 23 \mathrm{H} 3 \mathrm{~T})$ produced somewhat less severe effects. These data demonstrate that the nucleotides of the heptamer close to the cleavage site are critical for DSB formation in cis.

Mutations in the first and third positions of the heptamer also reduced DSB formation in trans to levels comparable with those of a substrate containing a single RSS (Figure 2A, compare $\mathrm{pNo} 12$ and $\mathrm{pNo} 23$ ). This suggests that these
A

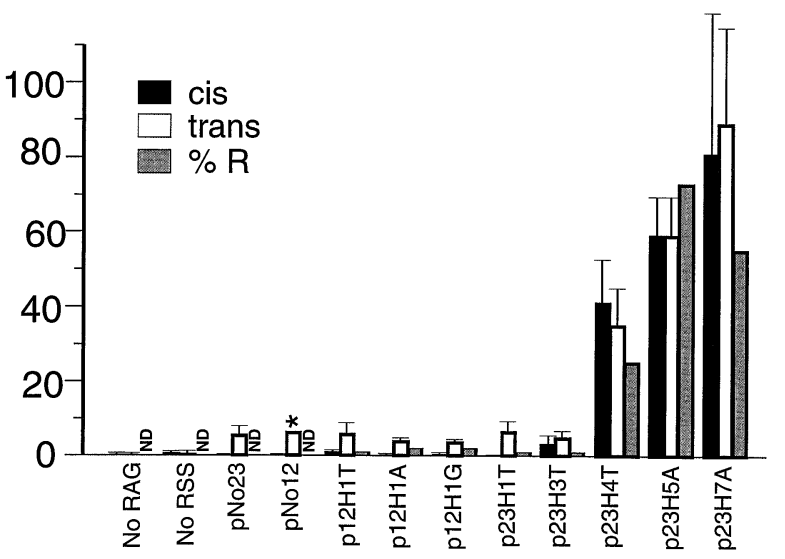

B

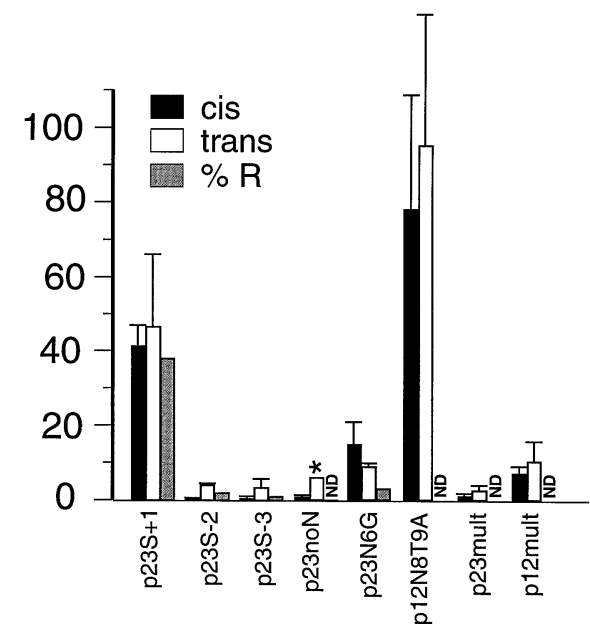

Fig. 2. Effects of RSS mutations on DSB formation in cis and in trans. (A) DSB levels derived from controls and from substrates containing heptamer mutations. (B) DSB levels derived from substrates containing point mutations in the spacer, nonamer or more than one RSS element. Plasmid names and mutations are explained in Table I. Levels were normalized to the abundance of DSBs derived from pJH290 (set at 100\%). Black bars, average levels of DSB in cis (at the mutant RSS); white bars, average levels of DSB in trans (at the consensus RSS). The data represent averages from at least three independent transfections of each mutant recombination substrate, and error bars indicate one standard deviation. The gray bars show the efficiency of formation of recombination products tested using substrates containing identical mutations and compared with the consensus 12/23 substrate, as assessed in a previous study (Hesse et al., 1989). Blots were probed either with end-labeled oligonucleotides or with a labeled PvuII fragment of pJH290 which gave identical results. Quantitation was performed with a phosphorimager and normalized to pJH290 levels from a parallel transfection. (*) For these two substrates, DSB levels in trans were only measured once in this study, but similar results have been reported previously from independent experiments (Steen et al., 1996).

positions are important for efficient DSB formation at the partner RSS. Mutations in the heptamer positions more distal to the cleavage site (p23H4T, p23H5A and p23H7A) only moderately reduced DSB levels in cis and in trans (Figure 2A).

Next we analyzed the effects of alterations in spacer length and nonamer sequence. As shown in Figure 2B, changing the spacer length by one nucleotide $(\mathrm{p} 23 \mathrm{~S}+1)$ only moderately reduced DSB formation both in cis and in trans. However, altering the spacer length by greater 


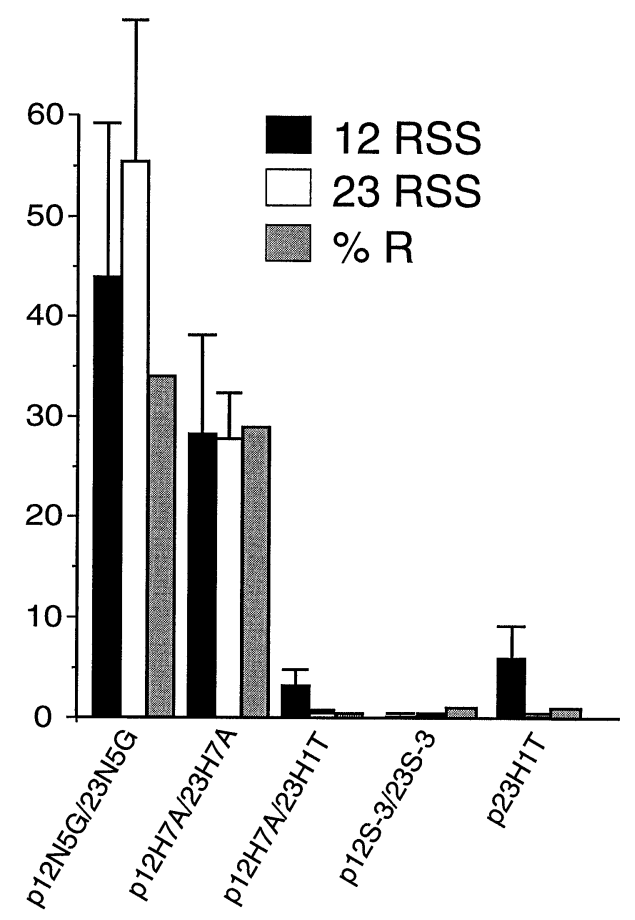

Fig. 3. Effects of multiple RSS mutations on DSB formation. LMPCR data from substrates containing mutations in both RSSs. Black bars, DSB levels at the 12-RSS; white bars, DSB levels at the 23-RSS; gray bars, efficiency of recombination as in Figure 2 (Hesse et al., 1989). DSB levels were measured for each substrate in at least three independent transfections, which were then averaged and normalized to pJH290 levels. Blots were probed and quantitated as described in Figure 2.

than one nucleotide (p23S-2 and p23S-3), like mutations in the first three positions of the heptamer, severely reduced cleavage in cis and in trans. Several different substrates containing altered nonamers were tested. Randomizing the nonamer sequence of the 23-RSS (p23noN) virtually eliminated DSB formation in cis and substantially reduced DSB in trans (Figure 2B). A different randomized nonamer sequence was also tested, with identical results (data not shown). Alteration of individual positions within the nonamer sequence also affected DSB levels although to a lesser degree. Changing the fifth position of the nonamer in both RSSs only had a moderate effect on the abundance of DSBs (Figure 3, p12N5G/23N5G). Simultaneously altering the last two nonamer positions also had little effect (Figure 2B, p12N8T9A). However, changing the sixth position significantly reduced levels of DSBs both in cis and in trans (Figure 2B, p23N6G).

Substrates containing multiple mutations in the RSS elements were also studied. Plasmid p12mult contains mutations in the heptamer and nonamer of the 12-RSS (H4T and N8T9A) which alone only slightly affect DSB formation. Together, these point mutations reduced cleavage in cis and in trans to levels substantially lower than observed with substrates containing the individual mutations (Figure 2B). This effect appears to be synergistic. Similarly, a substrate (p23mult) containing several moderate mutations in the heptamer (H6G, H7T), spacer $(\mathrm{S}+1)$ and nonamer (N5G) of the 23-RSS showed severely decreased DSB formation in cis and in trans (Figure 2B). Thus, even though individual changes in these positions have minor effects, the severe phenotype of RSSs con- taining multiple alterations indicates that these nucleotides nevertheless play important roles.

Point mutations in both RSSs also show at least a multiplicative effect on the abundance of signal ends. If each RSS contains an alteration that only slightly affects DSB formation, as in p12H7A/23H7A (Figure 3), DSB levels were reduced more than observed for the individual mutation (p23H7A, Figure 2A). In a substrate containing a moderate heptamer mutation in the 12-RSS and a severe mutation in the 23-RSS (p12H7A/23H1T), levels of DSBs at each RSS were substantially reduced (Figure 3). As expected, if each RSS contains a severe mutation (p12S$3 / 23 \mathrm{~S}-3$ ), no DSBs were observed (Figure 3 ). The additive effects of mutations in each RSS provide additional support for the notion that efficient cleavage requires an RSS pair.

Previous work has determined the effects of many of the same mutations studied here on the efficiency of recombination in vivo in a lymphoid cell line (Hesse et al., 1989). We find good agreement between these data (gray bars, Figures 2 and 3) and our measurements of effects of the heptamer, spacer and nonamer mutations on levels of DSBs. We also measured the effects of these mutations on efficiency of signal joint formation on the excised reciprocal product (diagrammed in Figure 1A) in fibroblasts using a quantitative PCR assay. This analysis of signal joint formation (in the same transfections used to measure DSBs) revealed excellent agreement between effects of mutations on cleavage and on joining of signal ends (data not shown). These results indicate that the primary effects of these mutations on the efficiency of recombination are exerted at or before the cleavage step, with little or no additional effects on joining.

\section{Dual and single RSS cleavage in vivo}

Although the LMPCR assay allows measurement of the effects of RSS mutations on DSB formation in cis and in trans, it does not distinguish between singly cleaved and doubly cleaved molecules. To study more directly the effects of RSS mutations on single and dual RSS cleavage, we developed a Southern blotting assay to measure these events. Southern analysis of undigested or PvuII digested DNA from transfection of pJH290 revealed a 328 bp fragment that corresponds to the expected length of an excised, doubly cleaved molecule terminating in signal ends (Figure 4B and C, lane 1). Due to its presence in undigested samples, this 328 bp species could represent either an excised linear molecule or an excised circular product containing a signal joint. Since the majority of signal joints retain all nucleotides of both RSSs, they form a diagnostic ApaL1 restriction site (verified by digestion of PCR products). The resistance of the $328 \mathrm{bp}$ fragment to ApaL1 (Figure 4B, lane 3) indicates that this species does not correspond to a circular molecule containing a signal joint. Furthermore, digestion with ClaI, which cleaves twice in the region between the two RSSs, did not produce the $102 \mathrm{bp}$ signal joint-containing fragment expected from a circular molecule (Figure 4C, lane 2). Finally, digestion with $D d e \mathrm{I}$, which cleaves once between the two RSSs, generated a pair of smaller fragments with sizes expected from digesting a linear molecule rather than producing the shift in fragment size expected from linearization of a circular molecule (Figure 4C, lane 3). 
A

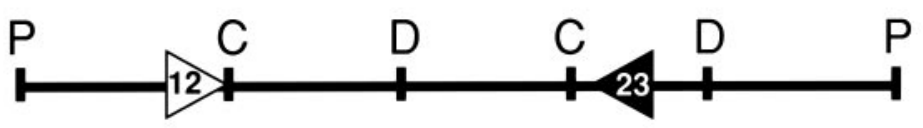

B

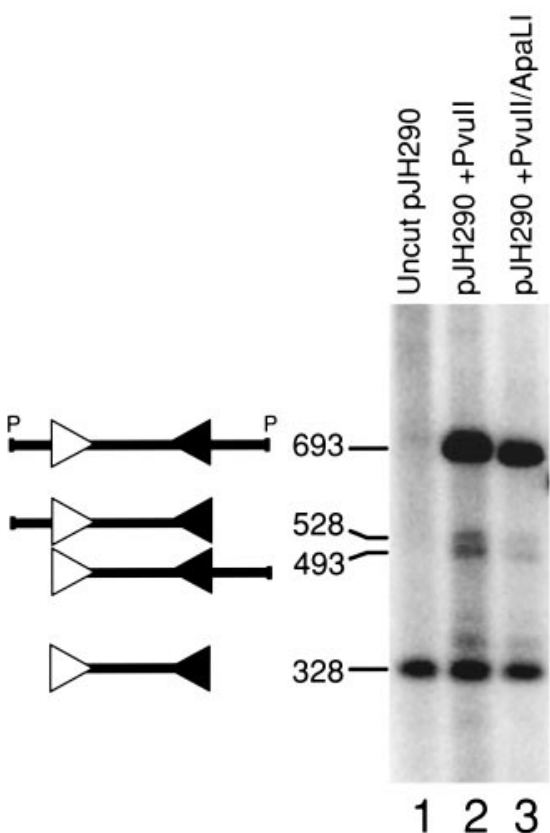

C

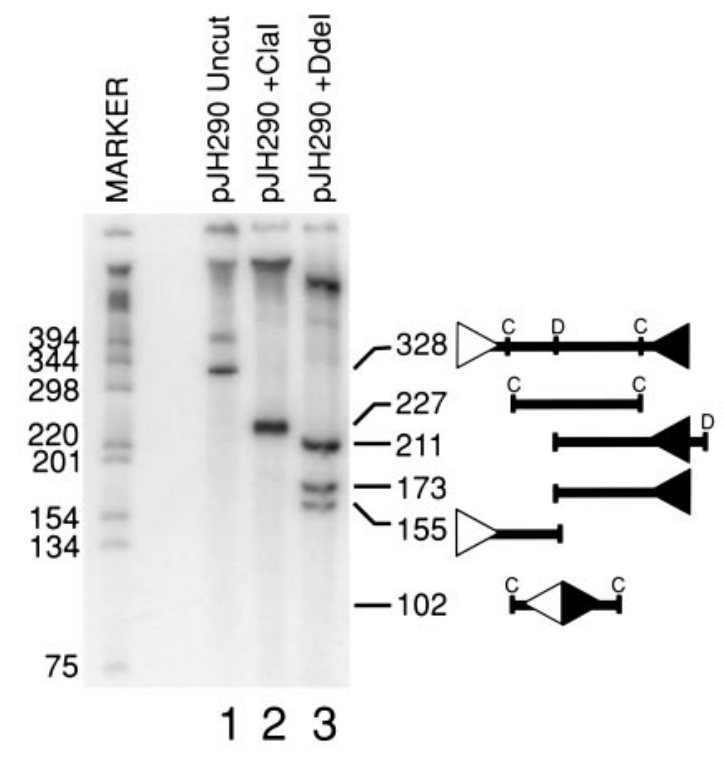

D

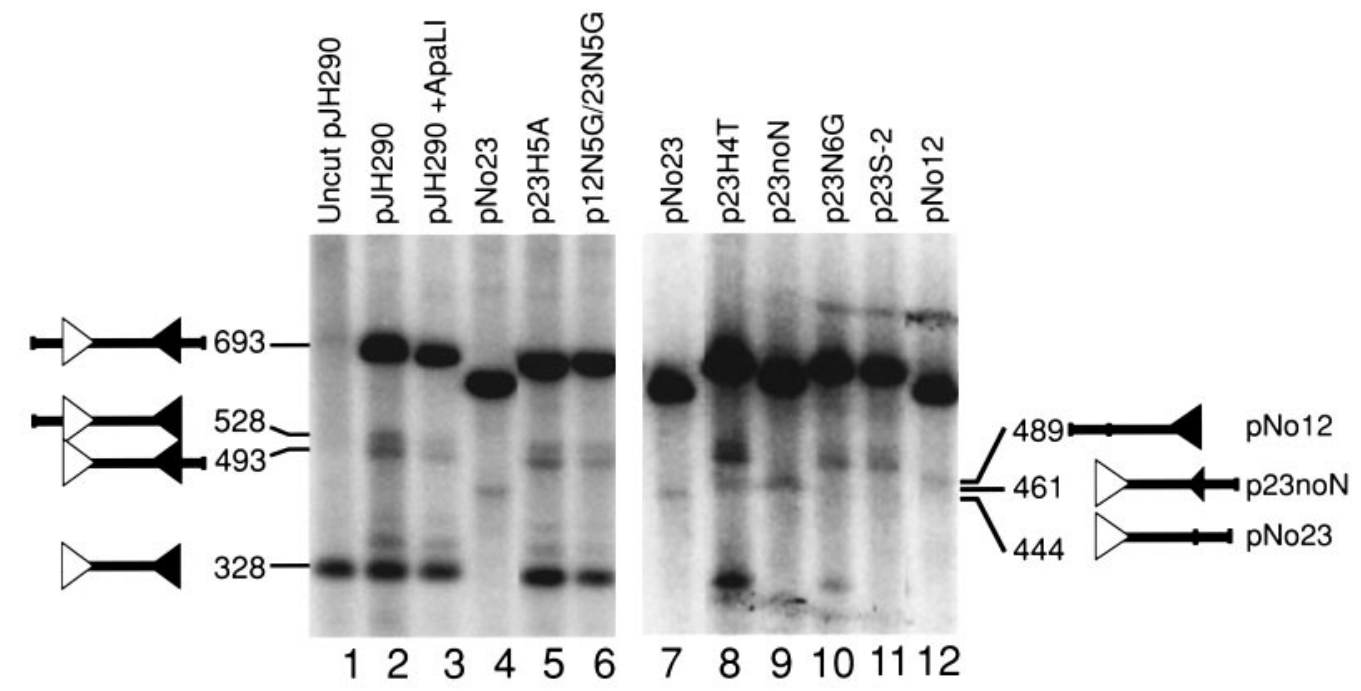

Fig. 4. Southern blotting analysis of recombination substrates. (A) A restriction map of the $693 \mathrm{bp} P v u I \mathrm{II}$ fragment of pJH290. Restriction sites: P, PvuII; C, ClaI; D, DdeI. (B) A Southern blot (1.8\% agarose gel) containing DNA from transfections of pJH290 that was uncut (lane 1), digested with PvuII (lane 2) or digested with PvuII and ApaL1 (lane 3). Sizes and identities of the molecular species: $693 \mathrm{bp}$, unrearranged fragment illustrated in (A); 528 bp, DSB only at the 23 RSS; 493 bp, DSB only at the 12-RSS; 328 bp, DSB at both RSSs. Large DNA species corresponding to unrearranged and singly cleaved plasmid in lane 1 are not resolved in this gel system and are not shown. The fragment migrating slightly slower than the 328 bp fragment (lanes 2 and 3) is the appropriate size for a coding joint product, an interpretation supported by other digests and hybridization with other probes (data not shown). (C) A Southern blot (6\% acrylamide gel) containing DNA from a transfection of pJH290 that was uncut (lane 1), digested with ClaI (lane 2), or digested with DdeI (lane 3). In lane 2, the 227 bp species corresponds to the ClaI fragment between the two RSSs in unrearranged or rearranged DNA; the expected position of the ClaI fragment containing the signal joint (102 bp) is also shown. In lane 3, the $211 \mathrm{bp}$ fragment results from DdeI digestion of unrearranged substrate; the $173 \mathrm{bp}$ and $155 \mathrm{bp}$ fragments result from digestion of the linear 328 bp molecule. (D) Southern blots (1.8\% agarose gels) of transfections of pJH290 or mutant recombination substrates. Two blots containing samples from two independent sets of transfections are shown. The first three lanes are the same as in (B). All lanes contain DNA digested with $P v u I I$ except lane 1 (uncut). Diagrams of molecular species with altered mobilities resulting from deletions in mutant substrates are indicated on the right. All blots were probed with a labeled $P v u I I$ fragment of pJH290 shown in (A). The small, dark triangle represents an RSS with a randomized nonamer; other symbols are as in Figure 1. 
Together, these data demonstrate that the 328 bp species corresponds to an excised linear molecule with RSS ends.

Direct detection of broken molecules by blotting allowed us to measure the relative abundance of DSBs. Quantitation of the blot shown in Figure $4 \mathrm{~B}$ revealed that the excised linear fragment is present at $15 \%$ of the level of the 693 bp unrearranged substrate fragment. In four separate transfections, the excised linear fragment was present at $22 \% \pm 10 \%$ of the abundance of the unrearranged substrate. Thus, we consistently observed conversion of a substantial fraction of the substrate to doubly cleaved molecules. Our inability to detect circular products containing signal joints by Southern blotting is consistent with data from PCR experiments which indicate that signal ends are $\sim 10$-fold more abundant than signal joints in these transfected samples (data not shown). This agrees with previous results from an Abelson leukemia virustransformed lymphoid cell line in which signal ends are substantially more abundant than signal joints under conditions allowing high expression of RAG proteins (Ramsden and Gellert, 1995).

The Southern blotting assay allows us to distinguish single and double RSS cleavage of extrachromosomal substrates for the first time. In addition to the $328 \mathrm{bp}$ excised linear fragment, $P v u I I$ digestion of DNA harvested from transfections of $\mathrm{pJH} 290$ revealed much less abundant species of $528 \mathrm{bp}$ and $493 \mathrm{bp}$, which are consistent with substrates containing DSBs at only the 23- or only the 12-RSS, respectively (Figure 4B, lane 2). Further evidence that these fragments are derived from single RSS cleavage was provided by additional mapping experiments (data not shown) and by analysis of substrates containing mutant RSSs (see below). The ability to detect single RSS cleavage events provides an opportunity to compare directly the levels of cleavage at the $12-\mathrm{RSS}$ and 23 -RSS in vivo. We find that the abundance of single DSBs at either of the two RSSs in pJH290 is roughly equivalent (Figure 4D, lane 2). Double and single RSS cleavage events were also analyzed in selected mutant substrates. As expected, no doubly cleaved molecules were detected using a substrate containing only a single 12-RSS (pNo23; Figure 4D, lanes 4 and 7) or a single 23-RSS (pNo12; Figure 4D, lane 12). However, in these substrates DSBs at the remaining RSS were present at levels roughly comparable with levels of single RSS cleavage in pJH290 (compare with lane 2; also data not shown). These data demonstrate that the levels of single RSS cleavage are not substantially affected by the presence of a second, consensus RSS.

Southern blotting analysis of other mutant substrates revealed effects on single and double RSS cleavage that are consistent with the LMPCR data presented in Figures 2 and 3. For example, RSS alterations that only moderately affected DSB formation, such as in p23H5A (lane 5), p12N5G/23N5G (lane 6) and p23H4T (lane 8), also exhibited only modestly reduced levels of dual RSS cleavage. As expected, these mutations also resulted in slight reductions in DSB levels at the mutant RSS. Substrates bearing RSS mutations that severely affected DSB formation, as measured by LMPCR, such as p23noN (lane 9) and p23S-2 (lane 11), showed substantially decreased levels of both single RSS cleavage in cis and dual RSS cleavage. However, low levels of DSBs in trans (at the consensus RSS) were observed, in agreement with the LMPCR data. Thus, severe mutations in the nonamer, spacer or heptamer elements appear to abrogate the ability of the RSS to stimulate cleavage in trans above the basal level observed in the absence of a second consensus RSS.

\section{Discussion}

A series of extrachromosomal recombination substrates was used to perform an extensive analysis of the effects of RSS mutations on cleavage both in cis and in trans. We found that certain mutations in each of the three RSS elements reduced the abundance of DSBs in cis (at the mutant RSS) by two orders of magnitude. These severe mutations also significantly decreased, but did not abolish, DSB formation in trans at a consensus partner RSS. Similar levels of single RSS cleavage were observed by Southern blotting at consensus RSSs in mutant substrates and in substrates containing a consensus 12/23 RSS pair. These data provide the first direct demonstration that cleavage can bypass the 12/23 rule in vivo, allowing cleavage to occur at a single RSS in substrates containing a 12/23 RSS pair. The close correlation between the effects of the RSS mutations studied here on the abundance of DSBs and on the efficiency of recombination suggests that these mutations do not have a major impact on the joining step of the reaction. In the following sections, these in vivo data are compared to and contrasted with recent results from cell-free systems.

\section{Components of the RSS required for DSB formation in cis}

Extensive in vitro analyses using a variety of oligonucleotide recombination substrates containing a single mutant RSS have shown that, while alterations in the first two positions of the heptamer did not affect the efficiency or accuracy of the first step of the cleavage reaction (introduction of nicks), the second step (conversion of nicks to DSBs) was blocked (Ramsden et al., 1996). Interestingly, either an alteration in the sixth position of the nonamer (N6G) or complete randomization of the nonamer sequence resulted in only a modest (7-fold) reduction in DSB formation. These data suggest that, under the conditions used (in $\mathrm{Mn}^{2+}$ ), the nonamer is not essential for cleavage.

Comparison of these results with our analysis of DSB formation in vivo reveals several similarities. As observed in vitro, the first nucleotide of the heptamer is critical for DSB formation, the third position is important, and altering positions 4-7 individually has only minor effects on cleavage in cis. Also in agreement with in vitro results, we found that alteration of the sixth position of the nonamer (N6G) or randomization of the nonamer significantly reduced DSB formation. However, in vivo the randomized nonamer has a substantially more severe effect than was observed in vitro, decreasing DSB in cis by another order of magnitude. This discrepancy is not due simply to the use of a different randomized sequence, as we obtained the same results with two randomized nonamers, including the sequence used in the cell-free experiments (Ramsden et al., 1996). Thus, our data suggest a role for nonamer residues in addition to N6 that was not revealed by in vitro studies. One possibility is that the RSS requirements for DSB formation in vivo are more 
stringent than have been observed in cell-free systems. This would suggest that some regulatory factor might be missing from the in vitro systems or that the conditions used in the cell-free reaction permit a partial bypass of the requirements for the nonamer.

Another significant difference between our in vivo data and previous in vitro results involves abundance of products produced by cleavage at the 12-RSS and the 23RSS. In vitro cleavage reactions reveal a substantial preference for cleavage of the 12-RSS under both relaxed and stringent reaction conditions (van Gent et al., 1995, 1996). Our direct detection of single RSS cleavage events by Southern blotting does not reveal a significant preference in vivo, either using substrates containing a 12/23 RSS pair or using substrates that contain only a single RSS. These data suggest that an additional factor not present in cell-free systems may be required for optimal cleavage at the 23-RSS.

\section{Components of the RSS required for DSB formation in trans}

Results from cell-free systems indicate that the major function of the $\mathrm{H} 1$ and $\mathrm{H} 2$ positions is in execution of the second chemical step, conversion of nicks to DSBs (Ramsden et al., 1996). These data raise the possibility that these two nucleotides might not be required for proper synaptic complex formation prior to cleavage. Precedents for this situation are found in other systems such as the Tn10 transposable element in which mutating the nucleotides involved in cleavage chemistry does not affect formation of a synaptic complex (Sakai et al., 1995). However, our analysis clearly demonstrates that alterations in the $\mathrm{H} 1$ and $\mathrm{H} 3$ positions severely decrease the abundance of DSB in trans. In fact, single nucleotide substitutions at either one of these positions affect DSB formation in trans as severely as removing the entire RSS. These data argue that mutations in the proximal nucleotides of the heptamer have effects both locally and at a distance, implying that these positions are important for synapsis or coordination of cleavage at both RSSs. In fact, none of the mutations studied here have discordant effects on DSB levels in cis and in trans. Thus, our data do not reveal separation of functions for the different elements of the RSS in vivo and suggest that both RSSs are important for efficient DSB formation, consistent with the requirement for synapsis.

\section{Single and dual RSS cleavage in vivo}

The experiments reported here indicate that efficient DSB formation in vivo requires a 12/23 RSS pair. However, we consistently detected low levels of DSBs in vivo using substrates containing only a single consensus RSS or a RSS pair containing one mutant RSS. These data indicate that the cleavage reaction can, rarely, bypass the requirement for proper synapsis, at least when substrates lack a 12/23 RSS pair. Our Southern blotting experiments clearly reveal that single RSS cleavage also occurs in substrates containing a 12/23 consensus RSS pair. The abundance of these singly cleaved molecules is remarkably high, $\geqslant 10 \%$ of the levels of doubly cleaved species, and is insensitive to the status (consensus or mutant) of the second RSS in the substrate. These data show that both in vivo and in vitro the recombination mechanism is capable of bypassing the requirement for a $12 / 23 \mathrm{RSS}$ pair. Although such single RSS cleavage events were not detected in our previous Southern blotting analysis of the TCR $\delta$ locus in mouse thymocytes (Roth et al., 1992b), a more sensitive analysis has recently found single RSS cleavage events at TCR $\delta$ at levels comparable with those we have observed in extrachromosomal substrates (Nakajima and Bosma, 1997).

Together, these data indicate that, under physiological conditions, single RSS cleavage events occur at surprisingly high levels. Although singly cleaved V(D)J recombination intermediates cannot go on to form standard junctions, they could participate in a variety of aberrant joining events. It is noteworthy that a class of nonstandard junctions termed 'open and shut' junctions, which can be explained by cleavage at a single RSS followed by processing and rejoining of the broken ends, occur frequently in both plasmid substrates and at endogenous antigen receptor loci (Lewis et al., 1988; Lewis and Hesse, 1991; Carroll et al., 1993; Fish and Bosma, 1994). Singly cleaved intermediates may also be more likely to participate in gross chromosomal rearrangements due to lack of an appropriate partner (Lewis and Hesse, 1991). It is not clear how these events bypass the normal requirement for synapsis. Understanding the mechanisms responsible for single RSS cleavage may provide insights into both the normal regulation of the cleavage reaction and abnormal rearrangement events that contribute to lymphoid neoplasia.

\section{Materials and methods}

\section{Recombination substrates}

Plasmid recombination substrates are based on pJH290 (Lieber et al., 1988), in which the RSSs are oriented so that recombination results in excision of the signal joint product. Some substrates were constructed in our laboratory; others were generous gifts of J.Hesse and M.Gellert (National Institutes of Health). The nucleotide sequences of all RSSs were confirmed by sequencing.

Expression vectors encoding full-length RAG-1 (pJH548), full-length RAG-2 (pJH549), truncated (core) RAG-1 (pMS127b; Sadofsky et al., 1993), and RAG-2 (pMS216; Sadofsky et al., 1994), generous gifts of M.Sadofsky, J.Hesse and M.Gellert (National Institutes of Health), were used as in our earlier study (Steen et al., 1996).

\section{Transient transfection assay}

Transient transfections were performed as described previously (Steen et al., 1996). Briefly, logarithmically growing CHO cells (CHO RMP41) were transiently transfected by the calcium phosphate method (CellPhect Transfection Kit, Pharmacia) with $2.1 \mu \mathrm{g}$ RAG-1 expression vector $2.5 \mu \mathrm{g}$ RAG-2 expression vector, and $5 \mu \mathrm{g}$ recombination substrate as described previously (Sadofsky et al., 1993; Steen et al., 1996). DNA was harvested $40-48 \mathrm{~h}$ after the transfection. Although the effects of RSS mutations on cleavage were similar with full-length and truncated RAG proteins, in agreement with our previous results (Steen et al., 1996), data from transfections using truncated RAG proteins are shown to facilitate comparison with in vitro results. All mutant recombination substrates were transfected in a minimum of three independent transfections and always in parallel with pJH290.

\section{LMPCR assay}

LMPCR was performed as described (Zhu and Roth, 1995; Steen et al., 1996). Briefly, ligations were performed overnight at $14^{\circ} \mathrm{C}$ with $2 \mathrm{U}$ of T4 DNA ligase (Gibco BRL), 200 pmol of annealed oligonucleotide primer DR19/20 (Roth et al., 1993), and 1/15th of the DNA harvested from a transfection. LMPCRs were performed in a PE 9600 thermal cycler (Perkin-Elmer) for 24 cycles as described (Roth et al., 1993; Zhu and Roth 1995; Steen et al., 1996) using primers DR20 (Roth et al., 1993) and either DR55 (5'-AGAGGGACTGGATTCCAAAGTTCTC) 
to detect the 12-RSS end or ML68 (5'-GCAACTTGTCGCGCCAATCG) to detect the 23 RSS end (Steen et al., 1996). Blots were probed with a random-primed, ${ }^{32} \mathrm{P}$-labeled 693 bp PvuII fragment of pJH290 containing the RSS pair or with ${ }^{32} \mathrm{P}$ end-labeled oligonucleotide probes DR55 (Steen et al., 1996) or DR66 (5'-GATCCTCTCATCGATGAGAGGATCC). Both types of probe gave similar results, only differing in sensitivity.

Quantitation of PCR products was performed using a Molecular Dynamics PhosphorImaging System. For each set of parallel transfections on a given blot, PCR product levels resulting from each substrate were assigned values by comparison to undiluted pJH290 DSB levels (set to $100 \%$ ).

\section{Southern blot assay}

DNA was digested with the appropriate restriction enzyme and subjected to electrophoresis. Blots were probed with the $693 \mathrm{bp} P v u \mathrm{II}$ fragment of pJH290 (see above).

\section{Acknowledgements}

We thank J.Hesse and M.Gellert for generously providing mutant substrates. We are grateful to T.Baker, J.Hesse and M.Gellert for many stimulating discussions. M.Bogue, J.-O.Han and S.Sazer made critical comments on the manuscript. M.Lowe provided secretarial assistance. This work was supported by a grant from the NIH (AI-36420). S.B.S. was supported by a National Institutes of Health Predoctoral Fellowship (T32-AI07495). D.B.R. is a Charles E.Culpeper Medical Research Scholar.

\section{References}

Akamatsu,Y., Tsurushita,N., Nagawa,F., Matsuoka,M., Okazaki,K., Imai,M. and Sakano,H. (1994) Essential residues in V(D)J recombination signals. J. Immunol., 153, 4520-4529.

Bogue,M. and Roth,D.B. (1996) Mechanism of V(D)J recombination. Curr. Opin. Immunol., 8, 175-180.

Carroll,A.M., Slack,J.K. and Mu,X.C. (1993) V(D)J recombination generates a high frequency of nonstandard TCR D $\delta$-associated rearrangements in thymocytes. J. Immunol., 150, 2222-2230.

Cuomo,C.A. and Oettinger,M.A. (1994) Analysis of regions of RAG-2 important for V(D)J recombination. Nucleic Acids Res., 22, 1810-1814

Cuomo,C.A., Mundy,C.L. and Oettinger,M.A. (1996) DNA sequence and structure requirements for cleavage of $\mathrm{V}(\mathrm{D}) \mathrm{J}$ recombination signal sequences. Mol. Cell. Biol., 16, 5683-5690.

Eastman,Q.M., Leu,T.M.J. and Schatz,D.G. (1996) Initiation of V(D)J recombination in vitro: the $12 / 23$ rule. Nature, 380, 85-88.

Fish,S.M. and Bosma,M.J. (1994) Abnormal deletions in the T cell receptor $\delta$ locus of mouse thymocytes. Mol. Cell. Biol., 14, 4455-4464.

Hesse,J.E., Lieber,M.R., Mizuuchi,K. and Gellert,M. (1989) V(D)J recombination: a functional definition of the joining signals. Genes Dev., 3, 1053-1061.

Kallenbach,S., Doyen,N., D’Andon,M.F. and Rougeon,F. (1992) Three lymphoid-specific factors account for all junctional diversity characteristic of somatic assembly of T-cell receptor and immunoglobulin genes. Proc. Natl Acad. Sci. USA, 89, 2799-2803.

Kirch,S.A., Sudarsanam,P. and Oettinger,M.A. (1996) Regions of RAG1 protein critical for V(D)J recombination. Eur. J. Immunol., 26, 886891.

Lewis,S.M. (1994) The mechanism of V(D)J joining: lessons from molecular, immunological and comparative analyses. Adv. Immunol., 56, 27-150.

Lewis,S.M. and Hesse,J.E. (1991) Cutting and closing without recombination in V(D)J joining. EMBO J., 10, 3631-3639.

Lewis,S.M., Hesse,J.E., Mizuuchi,K. and Gellert,M. (1988) Novel strand exchanges in V(D)J recombination. Cell, 55, 1099-1107.

Lieber,M.R., Hesse,J.E., Lewis,S., Bosma,G.C., Rosenberg,N., Mizuuchi,K., Bosma,M.J. and Gellert,M. (1988) The defect in murine severe combined immune deficiency: joining of signal sequences but not coding segments in V(D)J recombination. Cell, 55, 7-16.

McBlane,J.F., van Gent,D.C., Ramsden,D.A., Romeo,C., Cuomo,C.A., Gellert,M. and Oettinger,M.A. (1995) Cleavage at a V(D)J recombination signal requires only RAG1 and RAG2 proteins and occurs in two steps. Cell, 83, 387-395.

Nakajima,P.B. amd Bosma,M.J. (1997) Characterization of excised DNA intermediates associated with $\mathrm{V}(\mathrm{D}) \mathrm{J}$ recombination at the $\mathrm{T}$ cell receptor $\delta$ locus. Mol. Cell. Biol., in press.
Oettinger,M.A., Schatz,D.G., Gorka,C. and Baltimore,D. (1990) RAG-1 and RAG-2, adjacent genes that synergistically activate V(D)J recombination. Science, 248, 1517-1523.

Ramsden,D.A. and Gellert,M. (1995) Formation and resolution of doublestrand break intermediates in V(D)J rearrangement. Genes Dev., 9, 2409-2420.

Ramsden,D.A., McBlane,J.F., van Gent,D.C. and Gellert,M. (1996) Distinct DNA sequence and structure requirements for the two steps of $\mathrm{V}(\mathrm{D}) \mathrm{J}$ recombination signal cleavage. EMBO J., 15, 3197-3206.

Roth,D.B., Menetski,J.P., Nakajima,P.B., Bosma,M.J. and Gellert,M. (1992a) V(D)J recombination: Broken DNA molecules with covalently sealed (hairpin) coding ends in scid mouse thymocytes. Cell, 70, 983-991.

Roth,D.B., Nakajima,P.B., Menetski,J.P., Bosma,M.J. and Gellert,M. (1992b) V(D)J recombination in mouse thymocytes: Double-strand breaks near T cell receptor $\delta$ rearrangement signals. Cell, 69, 41-53.

Roth,D.B., Zhu,C. and Gellert,M. (1993) Characterization of broken DNA molecules associated with V(D)J recombination. Proc. Natl Acad. Sci. USA, 90, 10788-10792.

Sadofsky,M.J., Hesse,J.E., McBlane,J.F. and Gellert,M. (1993) Expression and V(D)J recombination activity of mutated RAG-1 proteins. Nucleic Acids Res., 21, 5644-5650.

Sadofsky,M.J., Hesse,J.E. and Gellert,M. (1994) Definition of a core region of RAG-2 that is functional in V(D)J recombination. Nucleic Acids Res., 22, 1805-1809.

Sakai,J., Chalmers,R.M. and Kleckner,N. (1995) Identification and characterization of a pre-cleavage synaptic complex that is an early intermediate in Tn10 transposition. EMBO J., 14, 4374-4383.

Schatz,D.G., Oettinger,M.A. and Baltimore,D. (1989) The V(D)J recombination activating gene, RAG-1. Cell, 59, 1035-1048.

Schlissel,M., Constantinescu,A., Morrow,T., Baxter,M. and Peng,A. (1993) Double-strand signal sequence breaks in V(D)J recombination are blunt, 5' -phosphorylated, RAG-dependent, and cell cycle regulated. Genes Dev., 7, 2520-2532.

Silver,D.P., Spanopoulou,E., Mulligan,R.C. and Baltimore,D. (1993) Dispensable sequence motifs in the RAG-1 and RAG-2 genes for plasmid V(D)J recombination. Proc. Natl Acad. Sci. USA, 90, 61006104.

Steen,S.B., Gomelsky,L. and Roth,D.B. (1996) The 12/23 rule is enforced at the cleavage step of $\mathrm{V}(\mathrm{D}) \mathrm{J}$ recombination in vivo. Genes to Cells, 1, 543-553.

van Gent,D.C., McBlane,J.F., Ramsden,D.A., Sadofsky,M.J., Hesse,J.E. and Gellert,M. (1995) Initiation of V(D)J recombination in a cell-free system. Cell, 81, 925-934.

van Gent,D.C., Ramsden,D.A. and Gellert,M. (1996) The RAG1 and RAG2 proteins establish the $12 / 23$ rule in V(D)J recombination. Cell, 85, 107-113.

van Gent,D.C., Hiom,K., Paull,T.T. and Gellert,M. (1997) Stimulation of V(D)J cleavage by High Mobility Group proteins. EMBO J., 16, 2665-2670.

Zhu,C. and Roth,D.B. (1995) Characterization of coding ends in thymocytes of scid mice: Implications for the mechanism of V(D)J recombination. Immunity, 2, 101-112.

Received on December 9, 1996; revised on January 20, 1997

\section{Note added in proof}

In support of our suggestion that an additional factor may be required for optimal cleavage of the 23-RSS, recent work demonstrates that addition of DNA-bending proteins HMG 1 or 2 to the in vitro cleavage reaction stimulates cleavage at the 23-RSS (van Gent et al., 1997). 